\title{
ON GEOMETRICAL PROPERTIES OF STARLIKE LOGHARMONIC MAPPINGS
}

\author{
ZAYID ABDUlhadi AND LAYAN EL HAJJ
}

\begin{abstract}
In this paper, we find the radius of the disk $\Omega_{r}$ such that every starlike logharmonic mapping $f(z)$ of order $\alpha$, is starlike in $|z| \leqslant r$ with respect to any point of $\Omega_{r}$. We also establish a relation between the set of starlike logharmonic mappings and the set of starlike logharmonic mappings of order $\alpha$. Moreover, the radius of starlikeness and univalence for the set of close to starlike logharmonic mappings of order $\alpha$ is determined.
\end{abstract}

Mathematics subject classification (2010): Primary 30C35, 30C45, Secondary 35Q30.

Keywords and phrases: Logharmonic mappings, rotationally starlike mappings, stable properties.

\section{REFERENCES}

[1] Z. Abdulhadi AND R. M. Ali, Univalent logharmonic mappings in the plane, Abstr. Appl. Anal. 2012, Art. ID 721943, pp. 1-32.

[2] Z. Abdulhadi, Close-to-starlike logharmonic mappings, Internat. J. Math. Math. Sci. 19 (1996), no. 3, 563-574.

[3] Z. Abdulhadi, Typically real logharmonic mappings, Int. J. Math. Math. Sci. 31 (2002), no. 1, 1-9.

[4] Z. Abdulhadi And Y. Abumuhanna, Starlike logharmonic mappings of order alpha, Journal of Inequalities in Pure and Applied Mathematics 7 (4) Art. 123, (2006), 1-6.

[5] Z. Abdulhadi And D. Bshouty, Univalent functions in $H \cdot \bar{H}(D)$, Trans. Amer. Math. Soc. 305 (1988), no. 2, 841-849.

[6] Z. Abdulhadi And W. Hengartner, Spirallike logharmonic mappings, Complex Variables Theory Appl. 9 (1987), no. 2-3, 121-130.

[7] Z. Abdulhadi, W. Hengartner and J. Szynal, Univalent logharmonic ring mappings, Proc. Amer. Math. Soc. 119 (1993), no. 3, 735-745.

[8] Z. Abdulhadi And W. Hengartner, One pointed univalent logharmonic mappings, J. Math. Anal. Appl. 203 (1996), no. 2, 333-351.

[9] Z. Abdulhadi and W. Hengartner, Polynomials in $H \bar{H}$, Complex Variables Theory Appl. 46 (2001), no. 2, 89-107.

[10] Y. Abu-Muhanna AND A. LyZZAiK, The boundary behaviour of harmonic univalent maps, Pacific J. Math. 141 (1990), no. 1, 1-20.

[11] M. Aydogan, Some results on a starlike log-harmonic mapping of order alpha, J. Comput. Appl. Math. 256 (2014), 77-82.

[12] M. Aydogan And Y. PolatoĞLu, A certain class of starlike log-harmonic mappings, J. Comput. Appl. Math. 270 (2014), 506-509.

[13] X. CHEN AND T. QIAN, Non-stretch mappings for a sharp estimate of the Beurling-Ahlfors operator, J. Math. Anal. Appl. 412 (2014), no. 2, 805-815.

[14] J. Clunie And T. Sheil-Small, Harmonic univalent functions, Ann. Acad. Sci. Fenn. Ser. A I Math. 9 (1984), 3-25.

[15] P. L. Duren, Univalent functions, Grundlehren der Mathematischen Wissenschaften 259, Springer, New York, 1983.

[16] P. Duren And G. SChober, A variational method for harmonic mappings onto convex regions, Complex Variables Theory Appl. 9 (1987), no. 2-3, 153-168. 
[17] P. Duren And G. SChober, Linear extremal problems for harmonic mappings of the disk, Proc. Amer. Math. Soc. 106 (1989), no. 4, 967-973.

[18] W. HengARTNER AND G. SCHOBER, On the boundary behavior of orientation-preserving harmonic mappings, Complex Variables Theory Appl. 5 (1986), no. 2-4, 197-208.

[19] W. Hengartner AND G. Schober, Harmonic mappings with given dilatation, J. London Math. Soc. (2) 33 (1986), no. 3, 473-483.

[20] S. H. JUn, Univalent harmonic mappings on $\Delta=\{z:|z|>1\}$, Proc. Amer. Math. Soc. 119 (1993), no. $1,109-114$.

[21] P. Li, S. Ponnusamy And X. WAng, Some properties of planar p-harmonic and log-p-harmonic mappings, Bull. Malays. Math. Sci. Soc. (2) 36 (2013), no. 3, 595-609.

[22] ZH. MAO, S. Ponnus Amy, AND X. WANG, Schwarzian derivative and Landau's theorem for logharmonic mappings, Complex Var. Elliptic Equ. 58 (2013), no. 8, 1093-1107.

[23] Z. NEHARI, The elliptic modular function and a class of analytic functions first considered by Hurwitz, Amer. J. Math. 69 (1947), 70-86.

[24] J. C. C. Nitsche, Lectures on minimal surfaces, vol. 1, translated from the German by Jerry M. Feinberg, Cambridge Univ. Press, Cambridge, 1989.

[25] R. Osserman, A survey of minimal surfaces, second edition, Dover, New York, 1986.

[26] H. E. ÖZKAN AND Y. POLATOĞLU, Bounded log-harmonic functions with positive real part, J. Math. Anal. Appl. 399 (2013), no. 1, 418-421. 